\title{
Primary tricuspid valve intimal sarcoma found in chest wall and lung tumors
}

\section{Akimasa Morisaki, Hiromichi Fujii, Yosuke Takahashi, Kokoro Yamane, Toshihiko Shibata}

\begin{tabular}{|c|l|}
\hline Citation & Asian Cardiovascular and Thoracic Annals. 28(2); 97-100 \\
\hline Issue Date & $2020-02-01$ \\
\hline Type & Journal Article \\
\hline Textversion & Author \\
\hline Rights & $\begin{array}{l}\text { The following article has been accepted by Asian Cardiovascular and Thoracic Annals } \\
\text { Vol.28, Issu.2, p.97-100. After it is published, it will be found at } \\
\text { https://oi.org/10.1177/0218492319875580. This article may be downloaded for } \\
\text { personal use only. Any other use requires prior permission of SAGE Publications. }\end{array}$ \\
\hline DOI & \begin{tabular}{l}
$10.1177 / 0218492319875580$ \\
\hline
\end{tabular} \\
\hline
\end{tabular}

Self-Archiving by Author(s)

Placed on: Osaka City University 


\section{Primary tricuspid valve intimal sarcoma found in chest wall and lung tumors}

Running head: Primary tricuspid valve intimal sarcoma

Akimasa Morisaki, MD, PhD*, Hiromichi Fujii, MD, PhD, Yosuke Takahashi, MD, PhD, Kokoro Yamane, MD, and Toshihiko Shibata, MD, PhD.

Department of Cardiovascular Surgery, Osaka City University Graduate School of Medicine, 1-4-3 Asahimachi, Abeno-ku, Osaka 545-8585Japan

*Corresponding Author: Akimasa Morisaki, MD, PhD

Department of Cardiovascular Surgery, Osaka City University Graduate School of Medicine 1-4-3 Asahimachi, Abeno-ku, Osaka 545-8585, Japan

Tel: +81-6-6645-3980; Fax: +81-6-6646-3071; email: m3_514@yahoo.co.jp

Declaration of conflicting interests: The author(s) declared no potential conflicts of interest with respect to the research, authorship, and/or publication of this article.

Funding: The author(s) have no financial support for the research, authorship, and/or publication of this article.

Manuscript Types: Case Study

Word count: 749 words, Reference counts: 4 


\begin{abstract}
A 50-year-old man with primary tricuspid valve intimal sarcoma and right ventricular and pulmonary arterial tumors with metastases to the chest wall and lung confirmed on plain radiographs initially presented with a cough. He underwent palliative complete removal of the cardiac tumor and partial removal of the right pulmonary arterial tumor with tricuspid valve replacement to avoid sudden death caused by tumor embolism and to definitively diagnose the cardiac tumor. The cardiac tumor arose from the tricuspid valve leaflets. Nine months postoperatively, the patient died secondary to fatal progression of the pulmonary arterial and lung tumors.
\end{abstract}

Keywords: intimal sarcoma; tricuspid valve; malignant cardiac tumor 


\section{Introduction}

Among cardiac sarcomas, intimal sarcomas are extremely rare, and appear most commonly in the pulmonary artery or aorta. ${ }^{1,2}$ We experienced a very rare patient with primary tricuspid valve intimal sarcoma with metastasis to the chest wall and lungs.

\section{Case report}

A 50-year-old man presented with a cough and rapidly-growing chest wall and lung tumors. He initially underwent $>3$ months of antibiotic therapy for pleuritis, at a nearby hospital. However, his cough and dyspnea persisted with worsening gradually, and follow-up chest Xrays detected the lung and chest wall tumors with pleural effusion. Computed tomographic angiography revealed a right pulmonary trunk tumor with a small left lower pulmonary arterial tumor, and a right ventricular tumor with floating and multiple chest wall and lung tumors (Fig. 1A-D). Needle biopsy and histopathology of one of the chest wall tumors confirmed a spindle cell tumor. At this point, he was referred to our hospital for treatment. Transthoracic echocardiography revealed a floating right ventricular tumor that arose from the tricuspid valve and subsequently extended to the pulmonary valve with pulmonary hypertension. Brain and bone imaging revealed no metastatic tumors. We diagnosed a primary malignant cardiac tumor with metastatic pulmonary arterial, lung, and chest wall tumors. We recommended, and the patient consented to, palliative surgery to relieve the symptom caused by cardiac tumor and the right pulmonary artery occlusion, and to prevent sudden death secondary to possible tumor embolisms from the cardiac tumor.

Under general anesthesia, we excised the combined tricuspid valve-right pulmonary arterial tumor and replaced the tricuspid valve through a median sternotomy. We established a conventional cardiopulmonary circuit with arterial and bicaval venous cannulations. Through a right atriotomy after cardiac arrest, we observed the botryoid tricuspid valve tumor with mucus production, arising from the posterior leaflet on the right ventricular side and the tricuspid annulus (Fig. 2). We removed the entire tumor, including noncancerous lesions, and replaced the tricuspid valve with a porcine bioprosthetic valve. Partial removal of the right pulmonary artery without endarterectomy was 
performed through a right pulmonary arterectomy in the right side of the superior vena cava because tumor invasion required lobectomy. The patient was extubated 2 hours postoperatively. After the operation, the dyspnea was dramatically improved. Histopathological findings revealed an intimal sarcoma of the tricuspid valve and pulmonary artery, based on the microscopic appearance and positive immunostaining for vimentin and negative staining for desmin, CD31, CD34, $\alpha$-actin, and Factor 8 (Fig. 3).

After discharge from our hospital, the patient underwent chemotherapy with adriamycin, as an outpatient. However, 9 months postoperatively (15 months from the initial onset of symptoms), the patient died secondary to progression of the pulmonary arterial and lung tumors, with no recurrent cardiac tumor.

\section{Discussion}

We reported an extremely rare case of primary tricuspid valve intimal sarcoma with pulmonary arterial, chest wall, and lung tumors. Most previously reported malignant cardiac tumors were angiosarcomas; however, based on autopsy findings, a recent report showed that intimal sarcoma was the most frequent malignant cardiac tumor, originating most often from the left heart, although intimal sarcoma is a commonly recognized mesenchymal tumor arising from the pulmonary artery or aorta. ${ }^{3}$ The report reclassified these tumors as intimal sarcomas, which were formerly classified as undifferentiated tumors, based on immunohistochemical analyses with overexpression and amplification of mouse/human double minute 2 protein (MDM2). Although we did not perform MDM2 immunohistochemistry in our patient, we diagnosed the cardiac tumor as an intimal sarcoma based on histopathology and positive vimentin immunostaining, which is distinct in mesenchymal cells, and negative desmin immunostaining, which is specific to muscle tissues. These findings differ from those for angiosarcoma and rhabdomyosarcoma.

Complete surgical tumor excision may improve prognosis in patients with malignant cardiac tumors; however, patients with unresectable cardiac tumors often die within months to 1 year. ${ }^{4}$ Cardiac tumors are most often identified secondary to symptoms caused by congestive heart failure or pulmonary hypertension, which are associated with advanced stage. Our patient had advanced cardiac 
intimal sarcoma with metastatic lesions in the lung and chest wall, which suggested an inoperable state. However, we performed cardiac tumorectomy with removing right pulmonary artery tumor and tricuspid valve replacement to relieve the symptom and prevent sudden death caused by possible tumor embolism. Postoperatively, the patient had no recurrence of the cardiac tumor following complete resection, but had fatal progression of the pulmonary arterial and metastatic tumors, despite chemotherapy, because these were unresectable lesions. Early diagnosis and complete resection of malignant cardiac tumors may be the only way to improve similar patients' prognoses.

Acknowledgement 


\section{References}

1. Burke A, Tavora F. The 2015 WHO Classification of Tumors of the Heart and Pericardium. $J$ Thorac Oncol. 2016;11:441-52.

2. Shah DK, Joyce LD, Grogan M, Aubry MC, Miller JA, Ding W, et al. Recurrent pulmonary intimal sarcoma involving the right ventricular outflow tract. Ann Thorac Surg. 2011;91:e41-2.

3. Neuville A, Collin F, Bruneval P, Parrens M, Thivolet F, Gomez-Brouchet A, et al. Intimal sarcoma is the most frequent primary cardiac sarcoma: clinicopathologic and molecular retrospective analysis of 100 primary cardiac sarcomas. Am J Surg Pathol. 2014;38:461-9.

4. Ramlawi B, Leja MJ, Abu Saleh WK, Al Jabbari O, Benjamin R, Ravi V, et al. Surgical treatment of primary cardiac sarcomas: Review of a single-institution experience. Ann Thorac Surg. 2016;101:698-702. 


\section{Figure Legends}

Figure 1. Preoperative computed tomography angiography findings. The white arrow indicates the cardiac tumor. The white broken arrow indicates the pulmonary arterial tumor. The wide and narrow outlined arrow indicates the metastatic lung and chest wall tumors, respectively. A, B, and C are axial images; D is an axial image of the lung window.

Ao, ascending aorta; PA, pulmonary artery; RA, right atrium; RV, right ventricle; RVOT, right ventricular outflow tract. 
Figure 1
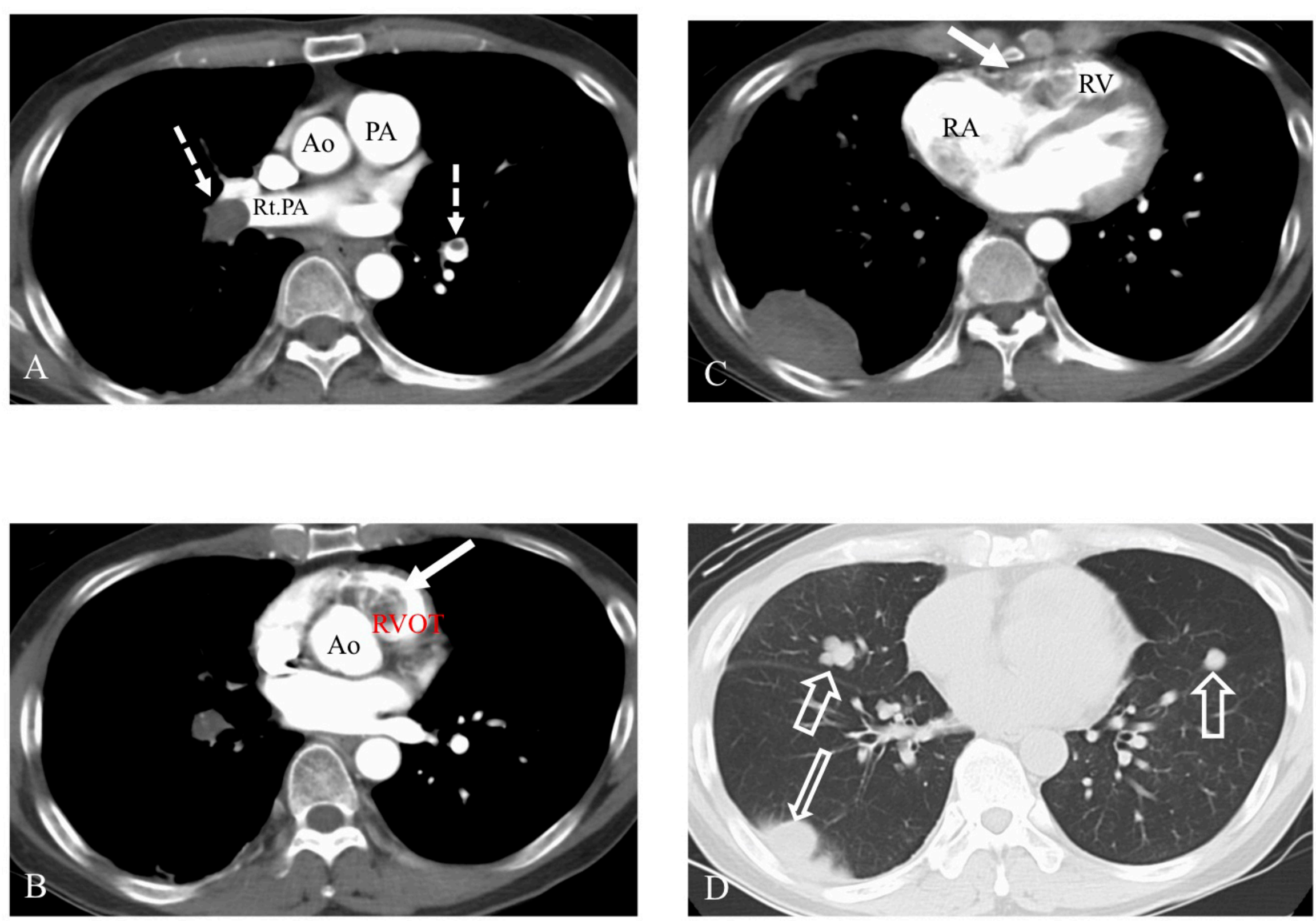
Figure 2. Intraoperative findings showing the botryoid tumor with mucus production, which arose from the posterior leaflet of the tricuspid valve and tricuspid annulus (white arrow).

RA, right atrium; SVC, superior vena cava; TV, tricuspid valve. 
Figure 2

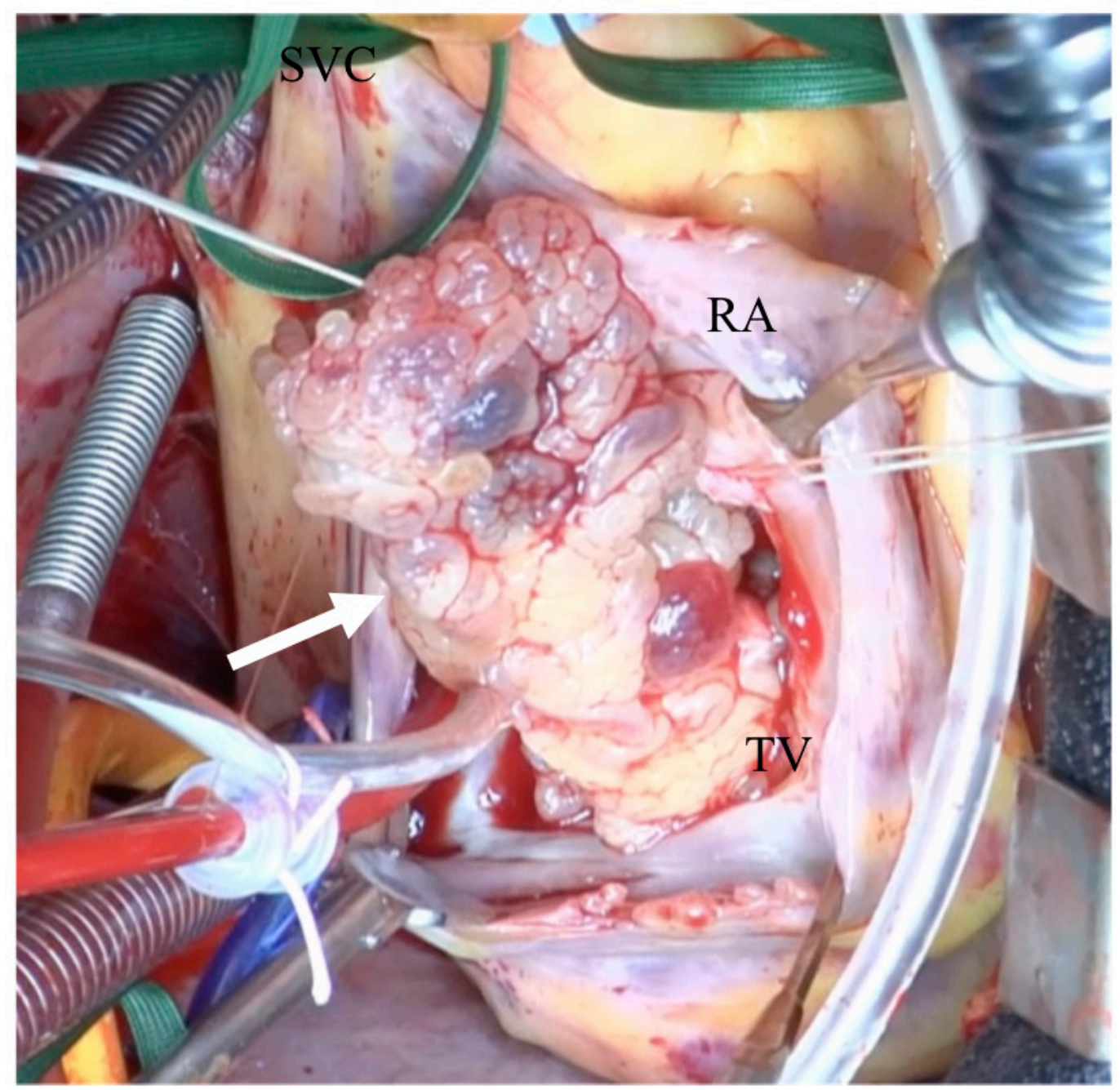


Figure 3. Histopathology of the cardiac tumor. A, Hematoxylin-eosin staining (magnification $\times 100$ ) showing central necrosis of the tumor with spindle cells on the surface of the tumor and myxomatous degeneration; B, Hematoxylin-eosin staining (magnification $\times 200$ ) showing highly-packed spindle cells with severe dyskaryosis and abnormal mitosis; C, Immunohistochemical profile showing positivity for antivimentin antibody and $\mathrm{D}$, negativity for antidesmin antibody. 


\section{Figure 3}

A

A
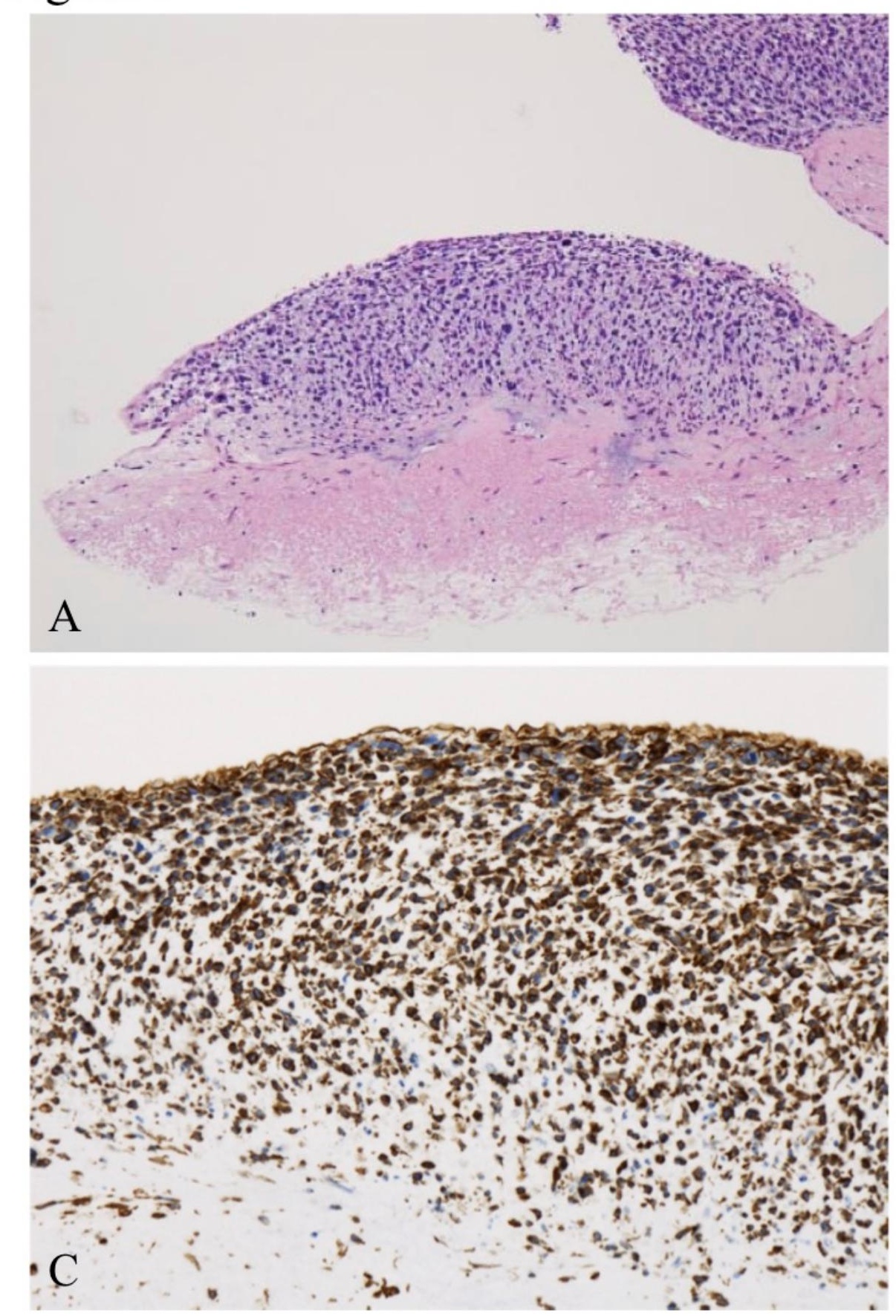
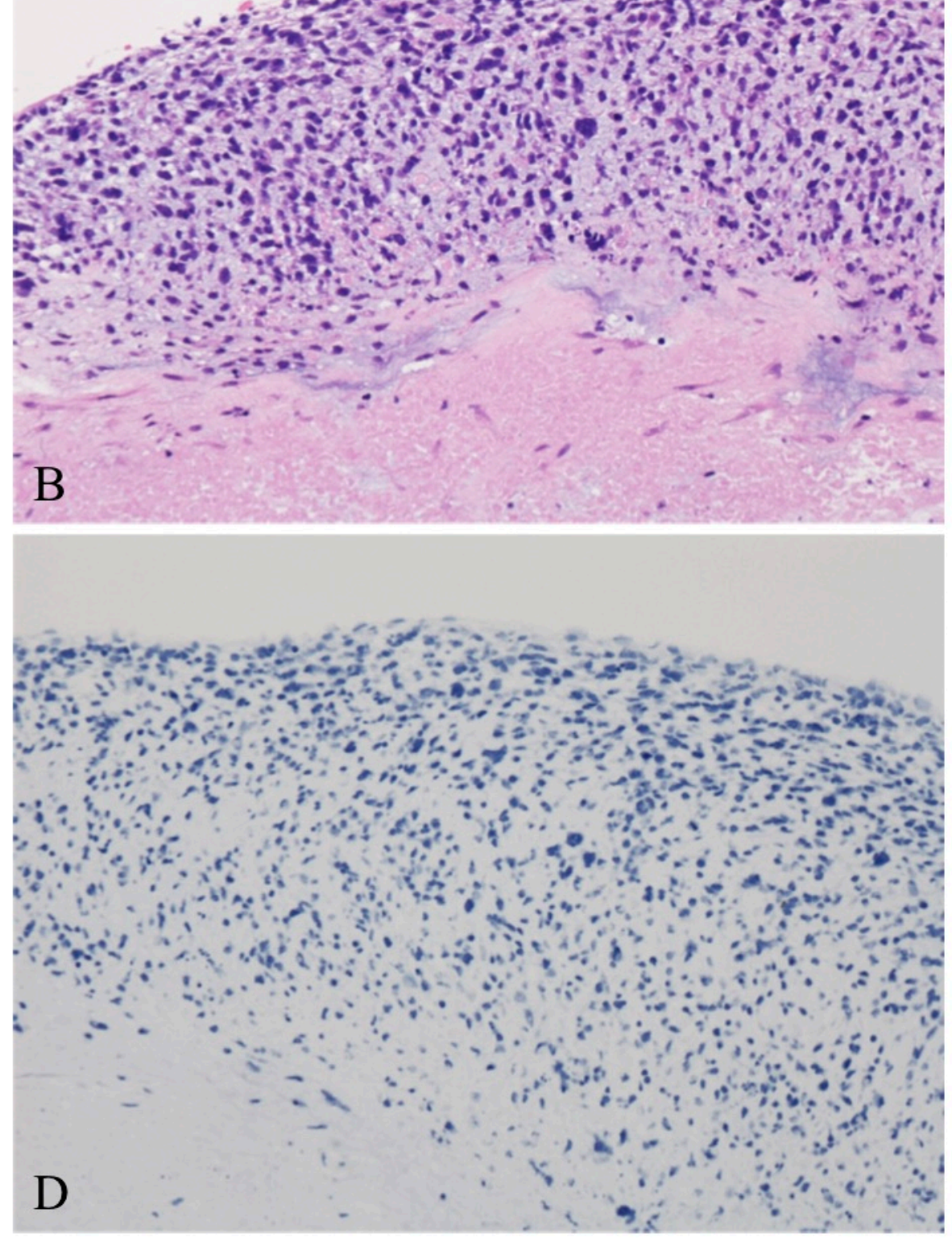